\title{
ESTRATEGIAS DE METACOMPRENSIÓN LECTORA Y ESTILOS DE APRENDIZAJE EN ESTUDIANTES UNIVERSITARIOS
}

\author{
Strategies of it puts understanding reader and styles of learning in university \\ students \\ Fanny Wong M. ${ }^{1}$, María Matalinares C. \\ Universidad Nacional Mayor de San Marcos, Lima, Perú \\ (RECiBido el 26/05/2011 - ACEPTADo el 27/06/2011)
}

\begin{abstract}
RESUMEN
La investigación que se presenta tiene como propósito establecer la relación de las estrategias de metacomprensión lectora con los estilos de aprendizaje en estudiantes universitarios. Los participantes fueron 809 estudiantes de ambos sexos a quienes se aplicó el Inventario de Estrategias de Metacomprensión de Schimitt y el Inventario de Estrategias de Aprendizaje de Kolb. Los resultados mostraron que los participantes poseen un bajo nivel de desarrollo de las estrategias metacomprensivas y no se evidencia un estilo de aprendizaje predominante. Finalmente, no se halló relación entre las estrategias de metacomprensión lectora y los estilos de aprendizaje.
\end{abstract}

Palabras clave: Estrategias de metacomprensión lectora, estilos de aprendizaje, estudiantes universitarios.

\begin{abstract}
The research presented aims to establish the relationship from reader's metacomprehension strategies with learning styles in college students. Participants were 809 students from both sexes who were applied the Strategies Inventory Schmitt metacomprehension of Inventory and Kolb's Learning Strategies. The results showed that participants have a low level of development strategies and no evidence a metacomprehension predominant learning style. Finally, no relationship was found between metacomprehension reading strategies and learning styles.
\end{abstract}

Keywords: Metacomprehension reading strategies, learning styles, college students. 


\section{INTRODUCCIÓN}

Al leer se produce la interacción texto-contexto-lector, en la que es el lector es quien finalmente crea el sentido, tiene consciencia de sus propios conocimientos y de su intención de lectura, pues aborda esta tarea con las estructuras cognitivas (conocimientos sobre la lengua y sobre el mundo) y afectivas (actitudes) que le son propias, al mismo tiempo que desencadena diferentes procesos que le permitirán comprender el texto, tales como los de elaboración e integración y las habilidades o procesos metacognitivos. Al respecto, para Giasson como también para Alvarado, con quienes concordamos, los procesos metacognitivos juegan un papel fundamental en la comprensión del texto y el recuerdo de eso que ha sido leído, facilitan la comprensión y permiten al lector ajustarse al texto y a la situación (Giasson, 1999 en Alvarado, 2003).

La metacognición es un concepto generado en el campo educativo principalmente durante experiencias de clase. Flavell fue el iniciador de su estudio y la explicó diciendo que "hace referencia al conocimiento de los propios procesos cognitivos, de los resultados de estos procesos y de cualquier aspecto que se relacione con ellos, es decir, el aprendizaje de las propiedades relevantes que se relacionen con la información y los datos. Por ejemplo, yo estoy implicado en la metacognición si advierto que me resulta más fácil aprender A que B" (Flavell, 1978, en Flavell, 1993). En consecuencia, la metacognición es un conocimiento de segundo grado, pero que va más allá, puesto que se implica en el proceso y regulación de los procesos de conocimiento. Es decir, tiene dos significados diferentes que se encuentran estrechamente vinculados: la metacoginición como producto y como proceso.

Como producto se da la metacognición cuando la vinculamos con el conocimiento que tenemos sobre nuestro funcionamiento cognitivo. Este es un conocimiento declarativo, "el saber qué". Por ejemplo, saber que extraer las ideas principales de un texto favorece la comprensión, saber que la organización de la información en un esquema facilita la comprensión. Como proceso, la metacognición alude al conocimiento de los procedimientos de supervisión y de regulación que implementamos sobre nuestra actividad cognitiva al enfrentar una tarea de aprendizaje. Este es un conocimiento procedimental: "saber cómo". Por ejemplo, saber seleccionar una estrategia para la organización de la información y estar en condiciones de evaluar el resultado obtenido (Chrobak, 2000).

Para Flavell, el control que una persona puede ejercer sobre su actividad cognitiva depende de las interacciones de cuatro componentes: conocimientos metacognitivos, experiencias metacognitivas, metas cognitivas y estrategias metacognitivas. Estas últimas revisten importancia en esta tesis. A diferencia de las estrategias cognitivas que se emplean para hacer progresar una actividad, las estrategias metacognitivas son las que se emplean para supervisar el proceso. La finalidad es lo que las determina. Por ejemplo, lectura y relectura son estrategias cognitivas y hacerse preguntas acerca de un texto leído para verificar la comprensión son estrategias metacognitivas porque van dirigidas a comprobar si se ha alcanzado la meta (Chrobak, 2000). Estrategias metacognitivas que, por otro lado, han sido clasificadas de diversas maneras (Schmitt, 1990).

En lo concerniente a la comprensión lectora, la metacognición recibe la denominación de metacomprensión lectora, por lo que se distinguen en ella los dos componentes 
metacognitivos: el primero, comprende los conocimientos que el sujeto lector posee sobre sus procesos cognitivos asociados con la lectura, vale decir, habilidades, estrategias y recursos con los que cuenta para tal fin, conocimiento que, por otro lado, demuestra que está comprometido activamente en un proceso de investigación, de comprensión y de autoevaluación. El segundo, es el de la regulación o del control cognitivo en la actividad de la lectura. El lector debe poner en práctica, como ya sabemos, mecanismos de control que guíen, planifiquen y verifiquen si la comprensión se efectúa eficazmente.

Así pues, un estudiante es cognitivamente maduro cuando sabe qué es comprender y cómo debe trabajar mentalmente para comprender. El desarrollo de la metacomprensión nos hace tomar conciencia por ejemplo, de que un párrafo es difícil de comprender y por eso controlamos la velocidad de lectura para, de esta manera, poder deducir el verdadero significado del escrito, con lo que el conocimiento de nuestra propia comprensión nos lleva a regular (autorregulación) la actividad mental implicada en la comprensión (Chrobak, 2000). En consecuencia, los procesos metacognitivos juegan un rol fundamental en la comprensión del texto y en el recuerdo de lo que ha sido leído, pues facilitan la comprensión y permiten al lector ajustarse al texto y al contexto (Vallés, 2005; Puente, 1994), destacando para el logro de estos propósitos, según Schmitt (1990), las siguientes estrategias metacognitivas: Predicción y verificación; pre-revisión (“vuelo de pájaro”), establecer un propósito; autointerrogatorio; uso de conocimientos previos; y, resumen.

De lo anterior, se deduce que el comprender una lectura no es develar el significado de cada una de las palabras ni siquiera de las frases, o de la estructura general del texto; sino más bien generar una representación mental del referente del texto, es decir, producir un escenario o modelo mental de un mundo real o hipotético en el cual el texto cobra sentido; producción en la que juega un papel decisivo la metacognición o metacomprensión lectora. Por tanto, el elevamiento de la comprensión lectora, pasaría por el elevamiento de la metacomprensión lectora; como lo indican estudios que evidencian la relación existente entre el entrenamiento en estrategias metacognitivas y el mejoramiento en la habilidad de comprensión de la lectura (Mateos, 2001).

Ahora bien, sobre estrategias metacognitivas Irrazabal, Saux, Burin y León (2006) realizaron una investigación cuyo propósito fue estudiar la relación entre la producción de resúmenes y la metacomprensión. Los resultados obtenidos indican, en primer lugar, diferencias significativas en los distintos componentes del resumen. Los alumnos mostraron un mejor desempeño al relevar los contenidos esenciales del texto que al producir una estructura que denote el sentido global del mismo. En conclusión, los resultados hallados ponen de manifiesto la necesidad de reforzar el entrenamiento en estrategias de metacomprensión que favorezcan la comprensión profunda y la posterior producción de textos consistentes.

Bravo Valdivieso, Villalón y Orellana (2006) efectuaron el estudio sobre los procesos cognitivos y el aprendizaje de la lectura inicial: diferencias cognitivas entre buenos lectores y lectores deficientes. Los autores encontraron una fuerte estabilidad en el rendimiento en lectura, apreciándose que los niños que ya traían a su ingreso al primer año ciertas bases prelectoras, como el conocimiento de las letras, el reconocimiento de nombres propios, días de la semana y conciencia fonológica eran mejores lectores durante todo el período estudiado. 
Anaya (2005) analizó los efectos del resumen sobre la mejora de la metacomprensión, la comprensión lectora y el rendimiento académico. A una muestra de 281 niños se les administró la Escala de Conciencia Metacognitiva de Lectura (ECML) y una Prueba de Comprensión lectora en un diseño pretest-postest con grupo de comparación. La variable dependiente fue la conciencia metacognitiva de la lectura y la comprensión lectora; la variable independiente fue el resumir los textos leídos, experiencia que duró cinco meses. Los resultados indican que el entrenamiento en la elaboración de resúmenes favorece de manera significativa a estos alumnos en cuanto a una mejora tanto de las habilidades metacomprensivas de la lectura, como de la comprensión lectora y el rendimiento académico.

Quaas, Ascorra y Bertoglia (2005) realizaron un estudio cuyo propósito fue determinar la relación entre los niveles de rendimiento de los alumnos de $8 .^{\circ}$ año de enseñanza básica de Valparaíso, Chile, en la asignatura de Lenguaje y Comunicación y las estrategias metacomprensivas de la lectura; mediadas estas últimas por el contexto de aprendizaje; es decir, por el clima social de aula y los métodos de enseñanza utilizados preferentemente por el profesor responsable de la asignatura en estudio. Los resultados obtenidos demostraron que existía relación entre las estrategias metacomprensivas de la lectura declaradas por los alumnos y el rendimiento escolar. Así mismo, se pudo establecer que el clima de aula y los métodos implementados por el profesor inciden en el desarrollo de las capacidades metacomprensivas lectoras.

Condori (2003) realizó el estudio experimental con la finalidad de mejorar la comprensión lectora de estos niños, aplicando las estrategias metacognitivas de predicción y verificación, revisión a vuelo de pájaro, establecimiento de propósitos y objetivos, autopreguntas, uso de conocimientos previos y, finalmente, resúmenes y aplicaciones de estrategias definidas. Los resultados demostraron que la aplicación de las estrategias mencionadas producen cambios en la forma de comprender e interpretar diferentes textos dentro de la tarea educativa.

Santibáñez y Pisconte (2003) realizaron una investigación que tuvo como propósito determinar la efectividad de un programa de reforzamiento de lectura orientado al desarrollo de habilidades metalectoras, en jóvenes tempranos que están cursando el segundo ciclo de estudios generales en la Universidad Nacional de Piura. La muestra estuvo constituida por 286 sujetos. Los resultados permitieron a los autores analizar y reflexionar no sólo sobre distintos ángulos de la competencia lectora, como parte de la competencia comunicativa dentro de las teorías de la lectura, sino también sobre la práctica pedagógica activa e intencional que la lectura, como fenómeno social, conlleva y que liga teóricamente el desarrollo con el pensamiento y este con el lenguaje en contextos determinados.

Bazán, Roldán y Villaroel (2003) desarrollaron una investigación con los propósitos de diagnosticar el nivel de Atención-Concentración y de Comprensión Lectora en los niños de cuarto y quinto grados de primaria de centros educativos estatales y el segundo, relacionar la capacidad de Atención-Concentración y nivel de Comprensión Lectora. Los hallazgos principales mostraron que los niños presentaron mejores niveles de comprensión lectora que las niñas. Los alumnos que presentaron niveles bajos de atención-concentración también presentaron niveles bajos de comprensión lectora, por el contrario a mayores niveles de atención-concentración también niveles altos de comprensión lectora. 
Ahora bien, al ser la metacognición conciencia o conocimiento que tiene que ver con el propio aprendizaje de las tareas que el aprendiz ha de realizar y con las estrategias que éste empleará para ello (Garner, 1987, en Puente, 1994), se deduce que, a su vez, es aprendida. Es decir, la metacognición puede ser analizada en el cómo se conforma o se aprende y, también, en su accionar como producto de esta conformación, en cómo influye sobre el aprendizaje (conocimiento, concientización, control y naturaleza de los procesos de aprendizaje). En este sentido, teniendo en cuenta que: a) el aprendizaje metacognitivo es una forma superior de aprendizaje, siguiéndole en jerarquía el aprendizaje cognitivo (memoria semántica, memoria episódica, conocimiento procedimental, conocimiento declarativo), el aprendizaje asociativo (condicionamiento operante, condicionamiento clásico) y el preasociativo (impronta, sensibilización, habituación) (Puente, 1994); y, b) comprendiendo por aprendizaje a "el proceso de adquisición de una disposición, relativamente duradera, para cambiar la percepción o la conducta como resultado de una experiencia” (Alonso, Gallego y Money, 1997 en Capella et al., 2003: 15), podemos decir que en el caso del aprendizaje metacognitivo la experiencia se elabora en el medio educativo en función de los estilos de enseñanza que se manifiestan a través de la presentación por el profesor de la materia o aspecto de enseñanza (Martínez, 1995, en Capella, et al., 2003), cuestión que se da en el proceso de enseñanza-aprendizaje, en el que, aparte del docente y del contenido, un rol decisivo lo juega el estudiante, las características de este, por ser quien en definitiva aprende en una experiencia muy personal. Por tanto, el aprendizaje metacognitivo puede ser desarrollado (o desalentado) mediante estilos de enseñanza y las características del estudiante adecuadas (o inadecuadas).

Por otro lado, con referencia a las características del estudiante que estarían implicadas en el aprendizaje metacognitivo, existen diferencias en cómo la mente procesa la información y cómo es influida por las percepciones del propio individuo, es decir las personas varían en sus estilos cognitivos o modos de percibir, recordar y pensar; tienen formas distintas de aprender, almacenar, transformar y emplear la información (Kogan, 1981, en Puente, 1994: 41), lo que tiene implicaciones educativas interesantes. Se han propuesto y estudiado varios estilos cognitivos, encontrándose entre los más importantes los siguientes: El estilo cognitivo Reflexividad-Impulsividad refiere a la manera cómo la persona afronta una situación problemática: reflexionando sobre esta o tendiendo a responder con rapidez y sin considerar las posibles alternativas. Una tendencia muy impulsiva trae como consecuencia una inhibición de la reflexión, lo cual, a su vez, impide el análisis de los conceptos. El estilo cognitivo Dependencia-Independencia del campo refiere al grado en que la persona percibe analíticamente. Los dependientes del campo perciben la información de manera global e influida por el contexto, prefieren mayor estructura externa, dirección e información de retorno (feedback) y están más a gusto con la resolución de problemas en equipo; por el contrario, los independientes del campo, tienden a percibir la información de manera analítica y menos determinada por el contexto, necesitando menos estructura externa e información de retorno, prefiriendo la resolución personal de los problemas y menos el aprendizaje en grupo (Puente, 1994; Capella, et al., 2003).

Ahora bien, los estilos de aprendizaje sirven como indicadores relativamente estables de cómo los estudiantes perciben, interaccionan y responden a sus ambientes de aprendizaje (Keefe, 1988, en Capella et al., 2003), y que conceptuamos siguiendo a Kolb (1976: 
21) como "algunas capacidades de aprender que se destacan por encima de otras como resultado del aparato hereditario, de las experiencias vitales propias y de las exigencias del medio ambiente actual", destacándose cuatro tipos dominantes de estilos de aprendizaje: Convergente, divergente, asimilador y acomodador. Caracterizando, por ejemplo, al primero, la aplicación práctica de las ideas; al segundo, la capacidad imaginativa; al tercero, la capacidad para crear modelos teóricos; y, al cuarto, la capacidad para hacer cosas.

En consecuencia, un elemento básico para la comprensión lectora es la metacognición o metacomprensión lectora, que es aprendida, y que puede ser estudiada analizándola en su conformación y en el cómo influye en el aprendizaje, jugando teóricamente un rol importante en su conformación los estilos de aprendizaje; en otras palabras, el que se empleen unas u otras estrategias metacognitivas estaría en función de estos estilos.

Además, no menos importante, los estilos de aprendizaje se ven influidos de alguna manera por las preferencias vocacionales y el desempeño laboral, según reportan estudios realizados en otros contextos socioculturales. Así, Plovnick en 1975, encontró al comparar la trayectoria académica de estudiantes de física que seguían una currícula que ponía énfasis en el aprendizaje convergente, que se presentaba una mayor deserción en los estudiantes de estilo divergente en comparación con los de estilo convergente, poniéndose de relieve que los estudiantes con un estilo menos adaptado a las exigencias académicas, tienden a dudar de la certeza de su elección de carrera y aun a desistir de la misma. Investigadores como Saavedra y Reynaldos (2006), Yacarini (2005), Delgado (2004) e Inga, García, Montes de Oca, Serpa, y Capa (2001) realizaron estudios con los estilos de aprendizaje en estudiantes universitarios.

\section{HIPÓTESIS}

Existen relaciones estadísticamente significativas entre las estrategias de metacomprensión lectora (Predicción y verificación; pre-revisión o "vuelo de pájaro", establecer un propósito; auto-interrogatorio; uso de conocimientos previos; y, resumen) y los estilos de aprendizaje (convergente, divergente, asimilador, acomodador) en los alumnos que estudian el primer año en una Universidad Nacional y una Universidad Privada.

\section{MÉTODO}

Para la realización de la presente investigación se empleó el tipo ex post facto correlacional, el diseño es no experimental del tipo transversal o transeccional.

La muestra de investigación estuvo conformada por 809 estudiantes de ambos sexos que cursaban el primer año en una Universidad Nacional y una Universidad Privada de Lima Metropolitana. Para la obtención de la muestra se aplicó un procedimiento de muestreo no probabilístico intencionado, dado el carácter de los variables a estudiar se utilizó: la técnica psicométrica y de observación indirecta. 


\section{INSTRUMENTOS}

Inventario de Estrategias de Metacomprensión (IEM). El inventario fue propuesto por Maribeth Schmitt (1990) El cuestionario fue traducido por Meza y Lazarte (2007). Está dividido en tres secciones, es decir, evalúa tres momentos en el proceso de la lectura. El primer momento consta de 10 ítems y en él se evalúan las estrategias metacomprensivas utilizadas antes de leer un texto. El Segundo momento también tiene 10 ítems, donde se evalúan las estrategias metacomprensivas utilizadas durante la lectura propiamente dicha de un texto. Y el último momento contiene 5 ítems orientados a evaluar las estrategias metas comprensivas utilizadas después de leer el texto.

Durante el primer momento, las estrategias que preferentemente se miden son las de predicción así como aquellas que ayudan a generar predicciones, como la revisión "a vuelo de pájaro" y la explicitación de conocimientos previos y las que ayudan a establecer los objetivos. Como no se ha empezado a leer el texto, es necesario planificar la lectura $\mathrm{y}$ tales estrategias ayudan a poderlo hacer.

Durante el segundo momento, las estrategias que se miden son las de verificación y en menor medida, las de auto-preguntas, explicitación de conocimientos previos, uso del resumen y estrategias definidas. Como se está efectuando la lectura, se requerirá supervisar si se está comprendiendo y si es necesario hacer reajustes.

Finalmente, durante la tercera sección, las estrategias que se miden implican una evaluación de las predicciones realizadas, de los objetivos establecidos en un comienzo, de los conocimientos previos explicitados y de los principales puntos del texto.

A cada ítem le acompañan 4 alternativas de respuesta. Solo una es la correcta, mientras que las demás quedan descartadas por las siguientes razones:

Constituyen elementos distractores, de manera que elegirlas indicaría que el evaluado no está realmente comprometido con la prueba o no sigue instrucciones o no atiende debidamente las alternativas, poniendo en riesgo la validez de sus respuestas. Tal es el caso del ítem 1, en el que la alternativa D se refiere a "pensar acerca de lo que ya se ha dicho en el texto hasta donde se ha leído", cuando todavía no se ha leído nada.

Se refieren a estrategias para mejorar la decodificación y no la comprensión. Dos ejemplos están dados por la alternativa B del ítem 4: "hacer una lista de las palabras de cuyo significado no estoy seguro(a) " y la alternativa A del ítem 19: "Probar si puedo reconocer las nuevas palabras del vocabulario".

Son estrategias o acciones secundarias o complementarias respecto de las centrales. Por ejemplo, "revisar si hubo suficientes figuras que ilustraran e hicieran interesante la lectura" (Alternativa B del ítem 21) puede ser útil para luego hacer predicciones con base en figuras pero no es lo más importante.

Tienen que ver con factores ambientales que condicionan el ejercicio de la lectura, como la alternativa D del ítem 10: "Determinar si tengo suficiente tiempo para realizar la lectura".

Se refieren a estrategias adecuadas para otros momentos de la lectura, pero no para el que se está evaluando "Leer el título para darme una idea sobre el contenido de la lectura" 
(Alternativa C del ítem 14) puede ser adecuado antes de empezar la lectura, pero no cuando ya se está leyendo.

Se trata de estrategias o acciones inadecuadas, que dificultan la comprensión antes que facilitarla. Leer sólo el comienzo y el final de un texto (señalado en la alternativa $\mathrm{C}$ del ítem 12), saltarse las partes más difíciles de un texto (indicado en el mismo ítem anterior), pensar en un mejor título para el material de lectura (señalado en la alternativa $\mathrm{D}$ del ítem 7) o pedir a alguien que le lea a uno el texto (indicado en la alternativa A del ítem 3) obstaculizan el proceso de extracción de significados (sobre todo, en los dos primeros casos), fomentan la pasividad mental (en especial, en el tercer caso) y traban el proceso de construcción de un modelo mental del texto, que es algo personal (sobre todo, en el último caso). Parecen ser irrelevantes, como "asegurarme que puedo pronunciar correctamente todas las palabras" (Alternativa C del ítem 7), "averiguar si los ejemplos son reales" (Alternativa A del ítem 16) o "revisar cuántas palabras del texto ya conozco" (Alternativa C del ítem 19).

La siguiente tabla muestra las puntuaciones que definen a cada uno de los niveles según la puntuación global alcanzada en el IEM.

Niveles de estrategias metacomprensión lectora (Puntuación global)

\begin{tabular}{cc}
\hline Puntuación & $\begin{array}{c}\text { Nivel de } \\
\text { Metacomprensión }\end{array}$ \\
\hline Menor a 13 & Nivel bajo \\
Entre 13 a 19 & Nivel medio \\
Mayor a 18 & Nivel alto \\
\hline
\end{tabular}

El Inventario de Estrategias de Metacomprensión Lectora (IEM) busca evaluar el conocimiento que los estudiantes de todos los niveles educativos tienen sobre las estrategias que deben utilizarse en la comprensión de lectura, antes, durante y después de hacerla. El IEM mide una variedad de estrategias metacomprensivas:

1. Predecir el contenido de un texto promueve su comprensión, brindándole al lector un propósito para leer. Verificar las predicciones ayuda a generar nuevas predicciones y a construir significados. Estas estrategias son evaluadas por los ítems 1, 4, 13, $15,16,18,23$.

2. Revisar a "vuelo de pájaro" un texto activa la experiencia previa del lector, proporcionándole información que le servirá para hacer predicciones. Es medida por los ítems 2 y 3.

3. Establecer propósitos u objetivos promueve la lectura activa y es medida por los ítems 5, 14 y 17.

4. Autopreguntarse promueve la comprensión activa y los ítems 6, 14 y 17 la miden. 
5. Explicitar los conocimientos previos (bagaje) aumenta el interés por la lectura y ayuda a generar predicciones. Es evaluada por los ítems 8, 9, 10, 19, 24 y 25.

6. Resumir sirve para monitorear la comprensión. Es evaluada por los ítems 11 y 22.

7. Releer o suspender la crítica y seguir leyendo, cuando se ha dejado de comprender, constituyen estrategias de lectura definidas. Son evaluadas por los ítems 12 y 20.

Todas estas estrategias son metacognitivas (relacionadas a la comprensión) porque demandan tomar conciencia de lo que acontece antes, durante y después de la lectura, permitiendo la rápida retroalimentación respecto de si se está yendo por buen camino o se debe efectuar correcciones para mejorar la comprensión. Cabe señalar que usualmente el énfasis estaba sobre lo que acontecía durante la lectura, pero las investigaciones en metacognición indican que el control de los tres momentos de una situación, facilita la comprensión.

La confiabilidad de la prueba se realizó mediante un análisis basado en el criterio de consistencia interna, se compararon las puntuaciones en el ítem del grupo que tiene las puntuaciones más altas en el inventario (y que por lo tanto tiene una mayor nivel de posesión de estrategias metacomprensivas), con las puntuaciones en el ítem del grupo que tiene las puntuaciones más bajas en el inventario (y, que por consiguiente, tiene un nivel bajo de posesión de estrategias metacomprensivas).

Los estadísticos descriptivos del puntaje total del IEM indican: media aritmética, 12,19; desviación estándar, 3,64; percentil 25: 10; percentil 75: 15. El grupo inferior está conformado por estudiantes con puntuaciones de 0 a 12, y el grupo superior por estudiantes con puntuaciones de 15 a 25. La aplicación de la prueba t de Student arrojó lo siguiente: 
Capacidad discriminativa de los ítems del IEM

\begin{tabular}{|c|c|c|}
\hline Ítem & $\mathrm{T}$ & $\begin{array}{c}\text { Nivel de significación } \\
\text { estadística }\end{array}$ \\
\hline 1 & 12,28 & 0,000 \\
\hline 2 & 7,53 & 0,000 \\
\hline 3 & 3,65 & 0,000 \\
\hline 4 & 10,47 & 0,000 \\
\hline 5 & 8,39 & 0,000 \\
\hline 6 & 11,26 & 0,000 \\
\hline 7 & 8,01 & 0,000 \\
\hline 8 & 14,58 & 0,000 \\
\hline 9 & 10,24 & 0,000 \\
\hline 10 & 7,90 & 0,000 \\
\hline 11 & 6,51 & 0,000 \\
\hline 12 & 0,45 & $0,648 * *$ \\
\hline 13 & 6,39 & 0,000 \\
\hline 14 & 9,40 & 0,000 \\
\hline 15 & 9,44 & 0,000 \\
\hline 16 & 9,82 & 0,000 \\
\hline 17 & 5,07 & 0,000 \\
\hline 18 & 7,58 & 0,000 \\
\hline 19 & 12,40 & 0,000 \\
\hline 20 & 5,41 & 0,000 \\
\hline 21 & 8,58 & 0,000 \\
\hline 22 & 7,97 & 0,000 \\
\hline 23 & 13,44 & 0,000 \\
\hline 24 & 10,31 & 0,000 \\
\hline 25 & 5,95 & 0,000 \\
\hline
\end{tabular}

Como se puede observar en la tabla, el único ítem que no tiene capacidad discriminativa es el 12, en tanto que todos los demás reactivos, según lo indica el nivel de significación correspondiente al valor $\mathrm{T}$ calculado, tienen esta capacidad, lo que significa que el inventario tiene características de ser consistente.

La Fiabilidad. Estimada con la técnica de la consistencia interna entendida como intercorrelación entre los ítems, utilizando el coeficiente alfa de Crombach. La estimación de la fiabilidad del IEM se realizó empleando el puntaje global. 
TABLA B. Fiabilidad del IEM $\left(\mathrm{N} .{ }^{\circ}\right.$ ítems $\left.^{2}=25\right)$

\begin{tabular}{ccc}
\hline $\begin{array}{c}\text { Media } \\
\text { aritmética }\end{array}$ & $\begin{array}{c}\text { Desviación } \\
\text { estándar }\end{array}$ & $\begin{array}{c}\text { Coeficiente alfa } \\
\text { de Crombach }\end{array}$ \\
\hline 12,19 & 3,63 & 0,61 \\
\hline
\end{tabular}

El coeficiente alfa de Crombach tiene un valor que es relativamente bajo, resultado que también ha sido observado en cuestionarios adaptados para su uso en nuestro país. Esta información unida a la buena capacidad discriminativa de los ítems nos permite indicar que el IEM tiene características de confiabilidad para medir las estrategias de metacomprensión aunque siempre con los recaudos que toda medición de variables psicológicas impone.

Validez. Se realizó un análisis factorial con la técnica de los componentes principales y rotación varimax con el criterio de Kaiser de retener los factores con un autovalor propio de 1 (García, Gil y Rodríguez, 2000). Antes de efectuar este análisis se comprobó la idoneidad de la matriz de correlaciones obteniéndose un índice KMO de 0,60 y un test de esfericidad de Bartlett estadísticamente significativo. Los resultados del análisis factorial indican que se obtienen 9 factores que explican el 48,28\% de la varianza de la matriz de correlaciones, valor que es considerado como aceptable. En resumen, estos datos apoyan el uso del IEM, pero a la vez sugieren que se debe continuar el análisis de su confiabilidad y de su validez estudiándolas en otras poblaciones con diferentes métodos y con relación a otras de sus fuentes.

Inventario de Estilos de Aprendizaje, construido por Kolb (1979), adaptado a nuestro medio por Escurra (1991), toma como base el modelo de aprendizaje experiencial. Este instrumento evalúa la preferencia por un determinado estilo de aprendizaje comparando los relativos predominios de una particular modalidad de aprender entre todas las posibles definidas por el modelo experiencial.

Es un inventario que puede ser aplicado tanto en forma individual como grupal, pudiendo incluso en algunos casos ser autoadministrado. Está constituido por 36 palabras, de las cuales 24 están asociadas a cada una de las cuatro fases o áreas del estilo de aprendizaje experiencial, las 12 palabras restantes son incluidas como elementos distractores para controlar la deseabilidad social de ahí que no sean utilizadas para el computo final. Evalúa las siguientes 4 áreas del aprendizaje experiencial:

- Experiencia Concreta (EC), implica que el sujeto debe ser capaz de poner énfasis en la interacción con otras personas en su vida cotidiana, pudiendo hacer un mayor uso de sus propias sensaciones que una aproximación sistemática al enfrentarse a los problemas que le toca vivir.

- Observación Reflexiva (OR), el sujeto debe poder entender las ideas y situaciones desde diferentes puntos de vista. Implica hacer uso de la objetividad y el juicio cuidadoso, utilizando los propios pensamientos y sentimientos para llegar a encontrar una opinión.

- Conceptualización Abstracta (CA), en esta capacidad se hace un prioritario uso de la lógica y las ideas para poder entender y comprender los problemas y situaciones 
que se presentan. Se utiliza mayoritariamente un planteamiento sistemático, así como el desarrollo de ideas y concepciones teóricas con la finalidad de poder resolver los problemas que se presentan.

- Experimentación Activa (EA). Se refiere a la capacidad de asumir un enfoque práctico con relación a los problemas, así como cierta preocupación respecto a la forma como realmente funcionan las cosas, sin tomar en cuenta la observación y el análisis de los problemas.

Estas áreas a su vez conforman las siguientes dimensiones: Concreto-Abstracta y Actividad Reflexión. Las respuestas deben ser presentadas por los sujetos ordenando jerárquicamente el grupo de 4 palabras, según el grado en el cual se percibe a la palabra que mejor caracteriza su estilo individual de aprender asignando puntajes de 1 a 4 , siendo este último para el más característica, el inventario tiene en total 9 grupos.

Producto de la interacción de las dos dimensiones básicas del aprendizaje, es factible reconocer la existencia de 4 tipos de estilo de aprendizaje:

- Convergente. En este estilo hay predominio de la conceptualización abstracta y de la experimentación activa.

- Divergente. Es el estilo en el cual se ha desarrollado más la experiencia concreta y la organización reflexiva.

- Asimilador. Es el estilo donde mayormente existe desarrollo de la conceptualización abstracta y de la observación reflexiva.

- Acomodador. Es el estilo caracterizado por el desarrollo de la experimentación activa y la experiencia concreta.

\section{Validez de contenido}

Los resultados del coeficiente $\mathrm{V}$ de Aiken indican que las palabras alcanzan coeficientes iguales o mayores que 0,80 , con lo que tienen una probabilidad asociada de 0,49 , por lo que todos los ítems son aceptados por los jueces, como muestras representativas de los estilos de aprendizaje y por ende es factible incluirlos en el instrumento, con lo que concluimos que el instrumento tiene validez de contenido.

\section{Validez de construcción}

Se procedió a establecer la relación entre las áreas y las dimensiones con el propósito de conocer la red nomológica. Es así que existen correlaciones estadísticamente significativas entre las áreas y sus dimensiones correspondientes: EC con CA-EC $(-0,853)$, entre OR con EA-OR (0.888), CA con CA-EC $(0,867)$ y EA con EA-OR $(0,880)$.

Los resultados permiten concluir que la red nomológica del IEA es válida y por lo tanto este instrumento posee validez de construcción. 


\section{Confiabilidad}

Los resultados del análisis de la confiabilidad realizados a través del método de consistencia interna y la homogeneidad, nos indica que tanto las áreas como las dimensiones alcanzan valores que oscilan entre el 0,67 y el 0,87, en el caso del coeficiente alfa de Cronbach y entre 0,75 y 0,89 con el coeficiente de castaño, esto permite considerar que IEA es un instrumento que tiene confiabilidad por consistencia interna.

\section{RESULTADOS}

A continuación se presentan los estadísticos descriptivos e inferenciales obtenidos en el análisis de los datos obtenidos.

TABLA 1. Alumnos por niveles de metacomprensión lectora

\begin{tabular}{lcc}
\hline Nivel de metacomprensión lectora & Frecuencia & Porcentaje \\
\hline Alto (20-25) & 18 & 2,20 \\
Medio (13-19) & 256 & 31,60 \\
Bajo (0-12) & 535 & 66,20 \\
\hline TOTAL & 809 & 100 \\
\hline $\begin{array}{l}\text { Prueba Ji Cuadrado de } \\
\text { homogeneidad = 496.630 }\end{array}$ & Grados de libertad $=2$ & $\begin{array}{c}\text { Significatividad }= \\
(\mathrm{p}<0.000) \text { Sí }\end{array}$ \\
\hline
\end{tabular}

Como se observa en la Tabla 1, los resultados de la prueba Ji cuadrado de homogeneidad, indican una diferencia estadísticamente significativa en todos los niveles de significación $(\mathrm{p}<0.000)$ entre las frecuencias de estudiantes clasificados en cada uno de los niveles de posesión de estrategias de metacomprensión lectora. El nivel prevalente es el Bajo, donde se ubican 535 alumnos (en otros términos 5 de cada 10) ingresantes a las Universidad Nacional y a la Universidad Privada. Por otro lado, también se destaca el ínfimo número de estudiantes en el nivel Alto de dichas estrategias: 18 ó el 2,22\% de 809 alumnos de la muestra. 
TABLA 2. Relación de la universidad de procedencia con el nivel global de las estrategias de metacomprensión lectora

\begin{tabular}{lccc}
\hline \multirow{2}{*}{ Metacomprensión lectora } & \multicolumn{2}{c}{ Universidad } & \multirow{2}{*}{ TOTAL } \\
\cline { 2 - 3 } & Nacional & Privada & \multirow{2}{*}{18} \\
\hline \multirow{2}{*}{ Alto (20-25) } & $9(2,0 \%)$ & $9(2,6 \%)$ & \\
Medio (13-19) & 0,6 & $-0,6$ & 256 \\
Bajo (0-12) & $136(38,6 \%)$ & $120(26,3 \%)$ & \\
& 3,8 & $-3,80$ & 535 \\
\hline TOTAL & $207(58,8 \%)$ & $328(71,8 \%)$ & 809 \\
\hline
\end{tabular}

TABLA 3. Resultados de la aplicación de la prueba Ji cuadrado de Independencia

\begin{tabular}{ccccc}
\hline $\begin{array}{c}\text { Valor Ji Cuadrado } \\
\text { de Independencia }\end{array}$ & $\begin{array}{c}\text { Grados de } \\
\text { libertad }\end{array}$ & $\begin{array}{c}\text { Nivel de } \\
\text { significación (a) }\end{array}$ & Significación & $\begin{array}{c}\text { Coeficiente V } \\
\text { De Cramer }\end{array}$ \\
\hline & 2 & 0,001 & $\begin{array}{c}\text { Valor Ji Cuadrado } \\
\text { estadísticamente } \\
\text { muy significativo }\end{array}$ & 0,136 \\
\hline
\end{tabular}

En la Tabla 3 se observa que la prueba Ji cuadrado de independencia tiene un resultado que es estadísticamente muy significativo $(\mathrm{p}<0.001)$, por lo tanto, los resultados observados en la Tabla 2 no son debido al azar, existiendo asociación, muy significativa entre la universidad de procedencia y los niveles de posesión de las estrategias de metacomprensión lectora, pero, a la vez, el tamaño del coeficiente $\mathrm{V}$ de Cramer indica que esta asociación, aun siendo muy significativa, no es fuerte. Por otro lado, los valores residuales estandarizados precisan las características de esta relación: En el nivel bajo de posesión de estrategias de metacomprensión lectora se encuentran más estudiantes de ambas universidades, pero los de la Universidad Privada se hallan significativamente en mayor número $71.8 \%$ o 7 de cada 10 de ellos) en comparación con los de la Universidad Nacional (58.8\% o 6 de cada 10 de ellos). En el nivel medio de posesión de las citadas estrategias se ubican de una manera estadísticamente significativa más estudiantes de la Universidad Nacional (38.6\% o 4 de cada 10 de ellos) en comparación con los alumnos de la Universidad Privada (26.3\% o 3 de cada 10 de ellos, aproximadamente). 
TABLA 4. Relación de la universidad con las estrategias de metacomprensión lectora

\begin{tabular}{|c|c|c|c|c|c|}
\hline Estrategia & Universidad & Media & $\begin{array}{l}\text { Desviación } \\
\text { estándar }\end{array}$ & $\begin{array}{l}\text { U de Mann- } \\
\text { Whitney (Z) }\end{array}$ & $\begin{array}{l}\text { Significación } \\
\text { estadística }\end{array}$ \\
\hline \multirow{2}{*}{$\begin{array}{l}\text { Predecir } \\
\text { (Puntaje teórico posible 0-4) }\end{array}$} & Nacional $(\mathrm{N}=352)$ & 1,92 & 1,11 & \multirow[b]{2}{*}{2,46} & \multirow[b]{2}{*}{ (p<0,03), Sí } \\
\hline & Privada $(\mathrm{N}=327)$ & 1,77 & 1,09 & & \\
\hline \multirow{2}{*}{$\begin{array}{l}\text { Verificar } \\
\text { (Puntaje teórico posible 0-3) }\end{array}$} & Nacional $(\mathrm{N}=352)$ & 1,39 & 0,90 & \multirow[b]{2}{*}{3,074} & \multirow[b]{2}{*}{$(\mathrm{p}<0,002)$, Sí } \\
\hline & Privada $(\mathrm{N}=327)$ & 1,19 & 0,86 & & \\
\hline \multirow{2}{*}{$\begin{array}{l}\text { Revisar } \\
\text { (Puntaje teórico posible } 0-2 \text { ) }\end{array}$} & Nacional $(\mathrm{N}=352)$ & 1,49 & 0,61 & \multirow[b]{2}{*}{0,520} & \multirow[b]{2}{*}{$(\mathrm{p}<0,603), \mathrm{NO}$} \\
\hline & Privada $(\mathrm{N}=327)$ & 1,8 & 0,70 & & \\
\hline \multirow{2}{*}{$\begin{array}{l}\text { Propósitos } \\
\text { (Puntaje teórico posible 0-3) }\end{array}$} & Nacional (N=352) & 1,70 & 0,92 & \multirow[b]{2}{*}{1,101} & \multirow[b]{2}{*}{$(\mathrm{p}<0.312), \mathrm{NO}$} \\
\hline & Privada $(\mathrm{N}=327)$ & 1,64 & 0,85 & & \\
\hline \multirow{2}{*}{$\begin{array}{l}\text { Autopreguntarse } \\
\text { (Puntaje teórico posible 0-3) }\end{array}$} & Nacional $(\mathrm{N}=352)$ & 1,69 & 0,99 & \multirow[b]{2}{*}{2,96} & \multirow[b]{2}{*}{ (p<0,003), Sí } \\
\hline & Privada $(\mathrm{N}=327)$ & 1,49 & 0,96 & & \\
\hline \multirow{2}{*}{$\begin{array}{l}\text { Extraer conclusiones } \\
\text { (Puntaje teórico posible 0-6) }\end{array}$} & Nacional (N=352) & 2,73 & 1,45 & \multirow[b]{2}{*}{3,06} & \multirow[b]{2}{*}{ (p<0,002), Sí } \\
\hline & Privada $(\mathrm{N}=327)$ & 2,40 & 1,38 & & \\
\hline \multirow{2}{*}{$\begin{array}{l}\text { Resumir } \\
\text { (Puntaje teórico posible 0-2) }\end{array}$} & Nacional $(\mathrm{N}=352)$ & 1,09 & 0,70 & \multirow[b]{2}{*}{0,908} & \multirow[b]{2}{*}{$(\mathrm{p}<0.364), \mathrm{NO}$} \\
\hline & Privada $(\mathrm{N}=327)$ & 1,03 & 0,74 & & \\
\hline \multirow{2}{*}{$\begin{array}{l}\text { Releer } \\
\text { (Puntaje teórico posible 0-2) }\end{array}$} & Nacional $(\mathrm{N}=352)$ & 0,70 & 0,76 & \multirow[b]{2}{*}{0,941} & \multirow[b]{2}{*}{$(\mathrm{p}<0,347), \mathrm{NO}$} \\
\hline & $(\mathrm{N}=327)$ & 0,76 & 0,68 & & \\
\hline
\end{tabular}

Los resultados de la prueba no paramétrica U de Mann Whitney mostrados en la Tabla 4 indican que, entre los estudiantes de las Universidades Mayor de San Marcos y Tecnológica del Perú existen diferencias en el empleo de las siguientes estrategias de metacomprensión lectora: predecir el contenido de un texto, verificar las predicciones, autopreguntarse y extraer conclusiones. En la primera estrategia la diferencia es estadísticamente significativa $(p<0.03)$, en las restantes es muy significativa $(p<0.01)$; y en todos los casos, la media aritmética más elevada es la del grupo de la Universidad de San Marcos. Los estudiantes de esta universidad muestran, si bien en un nivel predominantemente bajo, un mayor uso de las referidas estrategias. 
TABLA 5. Cantidad de alumnos por estilos de aprendizaje

\begin{tabular}{lcc}
\hline Estilo & Frecuencia & Porcentaje \\
\hline Divergente & 172 & 21,2 \\
Asimilador & 218 & 26,9 \\
Convergente & 222 & 27,4 \\
Acomodador & 197 & 24.4 \\
\hline TOTAL & 809 & 100 \\
\hline Prueba Ji Cuadrado de & \multirow{2}{*}{ Grados de libertad $=3$} & Significatividad $=$ \\
homogeneidad $=7.818$ & & $(\mathrm{p}<0.051) \mathrm{NO}$ \\
\hline
\end{tabular}

Se observa en la Tabla 5 los resultados de la prueba Ji cuadrado de homogeneidad que indican que no existe una diferencia estadísticamente significativa $(\mathrm{p}<0.051)$ entre las frecuencias de estudiantes clasificados en cada uno de los cuatro estilo de aprendizaje. A la muestra general de estudiantes universitarios del primer año no los caracteriza un estilo de aprendizaje específico.

TABLA 6. Relación de la universidad de procedencia con los estilos de aprendizaje

\begin{tabular}{lccc}
\hline \multirow{2}{*}{ Estilos de aprendizaje } & \multicolumn{2}{c}{ Universidad } & \multirow{2}{*}{ Total } \\
\cline { 2 - 3 } & Nacional & Privada & \\
\hline Divergente & $94(26,7 \%)$ & $78(17,1 \%)$ & 172 \\
Asimilador & $\mathbf{3 , 3}$ & $\mathbf{- 3 , 3}$ & \\
& $111(31.5 \%)$ & $107(23.4 \%)$ & 218 \\
Convergente & $\mathbf{2 , 6}$ & $\mathbf{- 2 , 6}$ & \\
& $69(19,6 \%)$ & $153(33.5 \%)$ & 222 \\
Acomodador & $\mathbf{- 4 , 4}$ & $\mathbf{4 , 4}$ & \\
\hline TOTAL & $78(22.2 \%)$ & $119(26 \%)$ & 197 \\
\hline
\end{tabular}

TABLA 7. Resultados de la aplicación de la prueba Ji cuadrado de Independencia

\begin{tabular}{ccccc}
\hline $\begin{array}{c}\text { Valor Ji Cuadrado } \\
\text { de Independencia }\end{array}$ & $\begin{array}{c}\text { Grados de } \\
\text { libertad }\end{array}$ & $\begin{array}{c}\text { Nivel de } \\
\text { significación }(\alpha)\end{array}$ & Significación & $\begin{array}{c}\text { Coeficiente V } \\
\text { de Cramer }\end{array}$ \\
\hline 28,735 & 3 & 0,000 & $\begin{array}{c}\text { Valor Ji Cuadrado } \\
\text { estadísticamente muy } \\
\text { significativo }\end{array}$ & 0,188 \\
\hline
\end{tabular}


Se observa en la Tabla 7 que el resultado de la aplicación de la prueba Ji cuadrado de independencia es estadísticamente muy significativo a todos los niveles de significación $(\mathrm{p}<0.000)$, por lo tanto, los resultados observados en la Tabla 6 no son debidos al azar, existiendo asociación muy significativa entre la universidad de procedencia y los estilos de aprendizaje, sin embargo, a la vez, el valor del coeficiente V de Cramer indica que esta asociación, aún siendo muy significativa no es fuerte.

Los valores residuales estandarizados precisan las características de esta relación: Estadísticamente, de una manera muy significativa, hay más estudiantes de San Marcos que de la Universidad Privada con un estilo de aprendizaje Divergente (26.7\% y $17.1 \%$, respectivamente).

Estadísticamente, de una manera muy significativa, hay más estudiantes de San Marcos que de la Universidad privada con un estilo de aprendizaje Asimilador (31.5\% y $23.4 \%$, respectivamente).

Estadísticamente, de una manera muy significativa - la significatividad más elevada de todas- hay más estudiantes de la Universidad Privada que en la Universidad Nacional con un estilo de aprendizaje Convergente (33,5\% y 19,6\%, respectivamente).

En el Estilo acomodador no se presentan diferencias estadísticas significativas entre los estudiantes de ambas universidades.

TABLA 8. Diferencias en las modalidades de aprendizaje, según Universidad

\begin{tabular}{|c|c|c|c|c|c|}
\hline Capacidades & Universidad & Media & $\begin{array}{c}\text { Desviación } \\
\text { estándar }\end{array}$ & $\begin{array}{l}\text { U de Mann- } \\
\text { Whitney (Z) }\end{array}$ & $\begin{array}{c}\text { Significación } \\
\text { estadística }\end{array}$ \\
\hline \multirow{2}{*}{$\begin{array}{l}\text { Experiencia concreta (EC) } \\
\text { (6 a } 24 \text { puntos) } \\
\text { (Media teórica: } 15)\end{array}$} & $\begin{array}{l}\text { Nacional } \\
(\mathrm{N}=352)\end{array}$ & 14,27 & 3,052 & \multirow{2}{*}{2,574} & \multirow{2}{*}{ (p $<0,01) ;$ SÍ } \\
\hline & $\begin{array}{c}\text { Privada } \\
(\mathrm{N}=327)\end{array}$ & 14,85 & 2,989 & & \\
\hline \multirow{2}{*}{$\begin{array}{l}\text { Conceptualización abstracta (CA) } \\
\text { ( } 6 \text { a } 24 \text { puntos) } \\
\text { (Media teórica: } 15)\end{array}$} & $\begin{array}{l}\text { Nacional } \\
(\mathrm{N}=352)\end{array}$ & 16,53 & 2,86 & \multirow{2}{*}{2,20} & \multirow{2}{*}{$(\mathrm{p}<0,03) ;$ SÍ } \\
\hline & $\begin{array}{c}\text { Privada } \\
(\mathrm{N}=327)\end{array}$ & 15,93 & 3,21 & & \\
\hline \multirow{2}{*}{$\begin{array}{l}\text { Observación reflexiva (OR) } \\
\text { (6 a } 24 \text { puntos) } \\
\text { (Media teórica: } 15)\end{array}$} & $\begin{array}{l}\text { Nacional } \\
(\mathrm{N}=352)\end{array}$ & 14,33 & 2,45 & \multirow{2}{*}{6,47} & \multirow{2}{*}{$(\mathrm{p}<0,000) ;$ SÍ } \\
\hline & $\begin{array}{c}\text { Privada } \\
(\mathrm{N}=327)\end{array}$ & 15,63 & 3,08 & & \\
\hline \multirow{2}{*}{$\begin{array}{l}\text { Experimentación activa (EA) } \\
\text { (6 a } 24 \text { puntos) } \\
\text { (Media teórica: } 15)\end{array}$} & $\begin{array}{l}\text { Nacional } \\
(\mathrm{N}=352)\end{array}$ & 15,08 & 2,50 & \multirow{2}{*}{1,77} & \multirow{2}{*}{$\begin{array}{c}(\mathrm{p}<0.07) \\
\text { NO }\end{array}$} \\
\hline & $\begin{array}{c}\text { Privada } \\
(\mathrm{N}=327)\end{array}$ & 14,68 & 3,37 & & \\
\hline
\end{tabular}


En la Tabla 8 se observa, en primer lugar que las medias aritméticas logradas por los dos grupos de estudiantes en las cuatro modalidades de aprendizaje, bordean un punto más o un punto menos a la media teórica (15); en segundo lugar, el resultado de la prueba no paramétrica U de Mann Whitney indican que entre los estudiantes de la Universidad nacional y la Universidad privada existen diferencias en las modalidades de aprendizaje Experiencia concreta, Conceptualización abstracta y Observación reflexiva, pero no en la modalidad de Experiencia activa. En la primera modalidad, la diferencia es estadísticamente muy significativa $(p<0,001)$, en la segunda la diferencia es estadísticamente significativa $(\mathrm{p}<0,03)$, y en la tercera, la diferencia es estadísticamente significativa en todos los niveles de significación $(\mathrm{p}<0.000)$. En Experiencia concreta y Observación reflexiva los estudiantes de la Universidad privada tienen la media aritmética más elevada; en Conceptualización abstracta, la media más alta corresponde a los estudiantes de la Universidad nacional.

TABLA 9. Relación del nivel global de las estrategias de metacomprensión lectora con los estilos de aprendizaje $(\mathrm{N}=809)$

\begin{tabular}{|c|c|c|c|c|c|}
\hline \multirow{2}{*}{$\begin{array}{l}\text { Nivel de estrategias } \\
\text { de metacomprensión } \\
\text { lectora (Global) }\end{array}$} & \multicolumn{4}{|c|}{ Estilos de aprendizaje } & \multirow{2}{*}{ Tota } \\
\hline & Divergente & Asimilador & Convergente & Acomodador & \\
\hline Alto (20-25) & $\begin{array}{c}(3.5 \%) \\
1,3\end{array}$ & $\begin{array}{c}5(2,3 \%) \\
0,1\end{array}$ & $\begin{array}{c}2(0,9 \%) \\
1,6\end{array}$ & $\begin{array}{c}5(2,5 \%) \\
-0,3\end{array}$ & 18 \\
\hline Medio (13-19) & $\begin{array}{c}56(32,6 \%) \\
0,3\end{array}$ & $\begin{array}{c}76(34.9 \%) \\
1,2\end{array}$ & $\begin{array}{c}62(27,9 \%) \\
-1,4\end{array}$ & $\begin{array}{c}62(31,5 \%) \\
-0,10\end{array}$ & 256 \\
\hline Bajo (0-12) & $\begin{array}{c}110(64 \%) \\
-0,7 \%\end{array}$ & $\begin{array}{c}137(62,8 \%) \\
1,2\end{array}$ & $\begin{array}{c}158(71,2 \%) \\
1,9\end{array}$ & $\begin{array}{c}130(66,0 \%) \\
0,0\end{array}$ & 535 \\
\hline Total & $172(100 \%)$ & $218(100 \%)$ & $222(100 \%)$ & $197(100 \%)$ & 809 \\
\hline
\end{tabular}

TABLA 10. Resultados de la aplicación de la prueba Ji cuadrado de Independencia

\begin{tabular}{ccccc}
\hline $\begin{array}{c}\text { Valor Ji cuadrado } \\
\text { de independencia }\end{array}$ & $\begin{array}{c}\text { Grados de } \\
\text { libertad }\end{array}$ & $\begin{array}{c}\text { Nivel de } \\
\text { significación }(\alpha)\end{array}$ & Significación & $\begin{array}{c}\text { Coeficiente V } \\
\text { de Cramer }\end{array}$ \\
\hline 6,137 & 6 & 0,408 & $\begin{array}{c}\text { Valor Ji Cuadrado } \\
\text { estadísticamente no } \\
\text { significativo }\end{array}$ & 0,06 \\
\hline
\end{tabular}

Se observa en la Tabla 10 que la prueba Ji cuadrado de independencia tiene un resultado que NO es estadísticamente significativo $(\mathrm{p}<0.408)$, por lo tanto, las diferencias en los resultados observados en la Tabla 11 son debidos al azar, NO existiendo asociación significativa entre los estilos de aprendizaje y el nivel global de posesión de las estrategias de metacomprensión lectora. Ambas variables son independientes. 
TABLA 11. Relación del nivel global de las estrategias de metacomprensión lectora con los estilos de aprendizaje, según la universidad de procedencia

\begin{tabular}{|c|c|c|c|c|c|c|}
\hline \multirow{2}{*}{ Universidad } & \multirow{2}{*}{$\begin{array}{c}\text { Nivel de estrategias } \\
\text { de metacomprensión } \\
\text { lectora (Global) }\end{array}$} & \multicolumn{4}{|c|}{ Estilos de aprendizaje } & \multirow{2}{*}{ Total } \\
\hline & & Divergente & Asimilador & Convergente & Acomodador & \\
\hline \multirow{4}{*}{ Nacional } & Alto & $4(4,3 \%) 1,2$ & $\begin{array}{c}3(2,7 \%) \\
0,10\end{array}$ & 0 & $\begin{array}{c}2(2,6 \%) \\
0,0\end{array}$ & 9 \\
\hline & Medio & $\begin{array}{c}34(36,2 \%) \\
-0,60\end{array}$ & $\begin{array}{c}41(36,9 \%) \\
-0,40\end{array}$ & $\begin{array}{c}25(36,2) \\
-0,50\end{array}$ & $\begin{array}{c}36(46,2 \%) \\
1,5\end{array}$ & 136 \\
\hline & Bajo & $\begin{array}{c}5659,6 \%) \\
0,20\end{array}$ & $\begin{array}{c}67(60,4 \%) \\
0,40\end{array}$ & $\begin{array}{c}44(63,8 \%) \\
0,90\end{array}$ & $\begin{array}{c}40(51,3 \%) \\
-1,5\end{array}$ & 207 \\
\hline & Total & 94 & 111 & 69 & 78 & 352 \\
\hline \multirow{4}{*}{ Privada } & Alto & $\begin{array}{c}54(69,2 \%) \\
-0,5\end{array}$ & $\begin{array}{c}70(65,4 \%) \\
-1,7\end{array}$ & $\begin{array}{c}114(74,5 \%) \\
0,90\end{array}$ & $\begin{array}{c}90(75,6 \%) \\
1,1\end{array}$ & 9 \\
\hline & Medio & $\begin{array}{c}22(28,2 \%) \\
, 4\end{array}$ & $\begin{array}{c}35(32,7 \%) \\
1,7\end{array}$ & $\begin{array}{c}37(24,2 \%) \\
-0,70\end{array}$ & $\begin{array}{c}26(21,8 \%) \\
-1,3\end{array}$ & 120 \\
\hline & Bajo & $\begin{array}{c}2(22,6 \%) \\
, 0.4\end{array}$ & $\begin{array}{c}2(1,9 \%) \\
-0,10\end{array}$ & $\begin{array}{c}, 2(1,3 \%) \\
-0,70\end{array}$ & $\begin{array}{c}3(2,5 \%) \\
0,50\end{array}$ & 328 \\
\hline & Total & 78 & 107 & 153 & 119 & 457 \\
\hline
\end{tabular}

TABLA 12. Resultados de la aplicación de la prueba Ji cuadrado de independencia

\begin{tabular}{lccccc}
\hline Universidad & $\begin{array}{c}\text { Valor Ji cuadrado de } \\
\text { independencia }\end{array}$ & $\begin{array}{c}\text { Grados de } \\
\text { libertad }\end{array}$ & $\begin{array}{c}\text { Nivel de } \\
\text { significación }(\alpha)\end{array}$ & Significación & $\begin{array}{c}\text { Coeficiente V } \\
\text { de Cramer }\end{array}$ \\
\hline UNMSM & 5,40 & 6 & 0,493 & $\begin{array}{c}\text { Valor Ji cuadrado } \\
\text { estadísticamente no } \\
\text { significativo }\end{array}$ & 0,09 \\
\hline UTP & 4,68 & 6 & 0,584 & $\begin{array}{c}\text { Valor Ji cuadrado } \\
\text { estadísticamente no } \\
\text { significativo }\end{array}$ & 0,07 \\
& & & & & \\
\hline
\end{tabular}

Los resultados de la prueba Ji cuadrado que se presentan en la Tabla 12 no son estadísticamente significativos, indicando que teniendo como variable control a la Universidad de procedencia no existe relación significativa entre los estilos de aprendizaje y las estrategias de metacomprensión de lectura (nivel Global). Las diferencias que se observan en la Tabla 12 se deben al azar, resultados que, por otra parte, confirman los presentados en la Tabla 9. 
TABLA 13. Relación de las estrategias de metacomprensión lectora con los estilos de aprendizaje $\left(\mathrm{N} .{ }^{\circ}=809\right)$

\begin{tabular}{|c|c|c|c|c|c|c|}
\hline $\begin{array}{l}\text { Estrategias de } \\
\text { Metacomprensión } \\
\text { lectora }\end{array}$ & $\begin{array}{l}\text { Estilos de } \\
\text { aprendizaje }\end{array}$ & N. ${ }^{\circ}$ & Media & $\begin{array}{l}\text { Desviación } \\
\text { típica }\end{array}$ & $\mathrm{F}$ & Significatividad \\
\hline \multirow{4}{*}{$\begin{array}{l}\text { Predecir } \\
\text { (Puntaje teórico posible } \\
0-4 \text { ) }\end{array}$} & Divergente & 172 & 1,81 & 1,09 & \multirow{4}{*}{1.042} & \multirow{4}{*}{$\begin{array}{c}(\mathrm{p}<0,373) \\
\quad \mathrm{NO}\end{array}$} \\
\hline & Asimilador & 218 & 1,88 & 1,09 & & \\
\hline & Convergente & 222 & 1,73 & 1,10 & & \\
\hline & Acomodador & 197 & 1,90 & 1,12 & & \\
\hline \multirow{4}{*}{$\begin{array}{l}\text { Verificar (Puntaje } \\
\text { teórico posible } \\
0-3 \text { ) }\end{array}$} & Divergente & 172 & 1,31 & 0,90 & \multirow{4}{*}{0,855} & \multirow{4}{*}{$(\mathrm{p}<0,464), \mathrm{NO}$} \\
\hline & Asimilador & 218 & 1,33 & 0,88 & & \\
\hline & Convergente & 222 & 1,20 & 0,86 & & \\
\hline & Acomodador & 197 & 1,28 & 0,90 & & \\
\hline \multirow{4}{*}{$\begin{array}{l}\text { Revisar (Puntaje teórico } \\
\text { posible } \\
0-2 \text { ) }\end{array}$} & Divergente & 172 & 1,50 & 0,59 & \multirow{4}{*}{1,062} & \multirow{4}{*}{$\begin{array}{c}(\mathrm{p}<0,365) \\
\quad \mathrm{NO}\end{array}$} \\
\hline & Asimilador & 218 & 1,44 & 0,62 & & \\
\hline & Convergente & 222 & 1,46 &, 58 & & \\
\hline & Acomodador & 197 & 1,54 &, 54 & & \\
\hline \multirow{4}{*}{$\begin{array}{l}\text { Propósitos (Puntaje } \\
\text { teórico posible } 0-3 \text { ) }\end{array}$} & Divergente & 172 & 1,76 & ,90 & \multirow{4}{*}{0,825} & \multirow{4}{*}{$\begin{array}{c}(\mathrm{p}<0,481) \\
\mathrm{NO}\end{array}$} \\
\hline & Asimilador & 218 & 1,63 & ,89 & & \\
\hline & Convergente & 222 & 1,63 & ,84 & & \\
\hline & Acomodador & 197 & 1,66 & ,90 & & \\
\hline \multirow{4}{*}{$\begin{array}{l}\text { Autopreguntarse } \\
\text { (Puntaje teórico posible } \\
0-3 \text { ) }\end{array}$} & Divergente & 172 & 1,75 & ,99 & \multirow{4}{*}{2,70} & \multirow{4}{*}{$\begin{array}{c}(\mathrm{p}<0,051) \\
\quad \mathrm{NO}\end{array}$} \\
\hline & Asimilador & 218 & 1,57 & 1,00 & & \\
\hline & Convergente & 222 & 1,47 & ,94 & & \\
\hline & Acomodador & 197 & 1,54 & ,97 & & \\
\hline \multirow{4}{*}{$\begin{array}{l}\text { Extraer } \\
\text { conclusiones (Puntaje } \\
\text { teórico posible 0-6) }\end{array}$} & Divergente & 172 & 2,56 & 1,45 & \multirow{4}{*}{0,739} & \multirow{4}{*}{$\begin{array}{c}(\mathrm{p}<0,529) \\
\text { NO }\end{array}$} \\
\hline & Asimilador & 218 & 2,64 & 1,44 & & \\
\hline & Convergente & 222 & 2,52 & 1,42 & & \\
\hline & Acomodador & 197 & 2,44 & 1,37 & & \\
\hline \multirow{4}{*}{$\begin{array}{l}\text { Resumir (Puntaje } \\
\text { teórico posible } \\
0-2 \text { ) }\end{array}$} & Divergente & 172 & 1,04 & 0,72 & \multirow{4}{*}{0,197} & \multirow{4}{*}{$\begin{array}{c}(\mathrm{p}<0,898) \\
\quad \text { NO }\end{array}$} \\
\hline & Asimilador & 218 & 1,05 & 0,72 & & \\
\hline & Convergente & 222 & 1,02 & 0,72 & & \\
\hline & Acomodador & 197 & 1,08 & 0,74 & & \\
\hline \multirow{4}{*}{$\begin{array}{l}\text { Releer (Puntaje teórico } \\
\text { posible } \\
0-2 \text { ) }\end{array}$} & Divergente & 172 & 0,72 & 0,62 & & \multirow{4}{*}{$\begin{array}{c}(\mathrm{p}<0,867) \\
\text { NO }\end{array}$} \\
\hline & Asimilador & 218 & 0,73 & 0,66 & & \\
\hline & Convergente & 222 & 0,77 & 0,66 & 0,243 & \\
\hline & Acomodador & 197 &, 7259 & 0,65 & & \\
\hline
\end{tabular}

Todos los resultados de la aplicación de la prueba $\mathrm{F}$ (análisis de varianza de una vía) que se presentan en la Tabla 13 son estadísticamente NO significativos, indicando que los 
estudiantes universitarios del primer año que participan de la investigación y que tienen diferentes Estilos de aprendizaje no se diferencian en el empleo de las estrategias de metacomprensión lectora: Predecir el contenido de un texto, verificar las predicciones, revisar a "vuelo de pájaro" un texto, establecer propósitos u objetivos, autopreguntarse, extraer conclusiones, resumir y releer.

\section{DISCUSIÓN}

Empezamos esta sección haciendo una breve síntesis de los resultados: a) el nivel global de posesión de las estrategias de metacomprensión que predomina en la muestra investigada es el bajo (véase Tabla 1); asimismo, en este nivel hay más alumnos de la Universidad Privada, mientras que en el nivel medio hay más alumnos de la Universidad Nacional (véase Tabla 2); b) consideradas las estrategias de metacomprensión lectora de manera individual, los estudiantes de la Universidad Privada, aunque con promedios ligeramente por debajo de los promedios teóricos, tienen un mayor rendimiento que los de la Universidad privada en las estrategias predecir, verificar, extraer conclusiones y autopreguntarse (véase Tabla 3); c) en la muestra total de estudiantes no hay un estilo de aprendizaje que predomine, pero consideradas por separado los grupos de estudiantes, los de la Universidad Nacional, se muestran más divergentes y asimiladores; mientras que los de la Universidad Privada son más convergentes (véase Tablas 6 y 7) ; d) por otro lado, en las modalidades o capacidades de aprendizaje, los estudiantes de la Universidad Nacional tienen un mayor rendimiento en la conceptualización abstracta (CA) y los de la Universidad Privada lo tienen en la experiencia concreta (EC) y la observación reflexiva (OR), no diferenciándose ambos grupos en la modalidad experimentación activa (véase Tabla 8); por último, e) los datos no respaldan la hipótesis de la existencia de relación entre las estrategias de metacomprensión lectora y los estilos de aprendizaje (Tablas 9,11 y 13).

El hallazgo de un nivel bajo de estrategias de metacomprensión lectora en los estudiantes universitarios analizados no es, en verdad, sorprendente pues si asumimos que estas estrategias se relacionan con la comprensión lectora (Condori, 2003; Irrazabal et al. 2006; Anaya, 2005; Santibáñez y Pisconte, 2003), estas tendrán que ser de ese nivel si prestamos atención a los magros resultados en las pruebas de comprensión lectora alcanzados por los estudiantes de secundaria en las investigaciones del PISA, los estudiantes de primaria en las mediciones del LLECE (Torreblanca, 2001) y estudiantes universitarios (González, 1998). Así pues, los resultados encontrados constituyen una constatación de una realidad no halagüeña. El que los estudiantes de la Universidad Nacional tengan en este nivel bajo un rendimiento más elevado que los de la Universidad Privada en algunas de las estrategias se explicaría por el examen de admisión más exigente al que son sometidos, examen que, sin embargo, también muestra sus debilidades en cuanto a las habilidades relacionadas con la comprensión lectora que evalúa el haber sido aprobado por estudiantes con esta característica. Pensamos que lo más conveniente ante esta realidad es iniciar o reforzar el trabajo de estas estrategias en los estudiantes universitarios de manera organizada, pues es factible su mejora como lo indican varios estudios (Condori, 2003; Anaya, 2005; Santibáñez y Pisconti, 2003), a la vez, es indispensable para esta mejora el trabajar estas estrategias por medio del desarrollo de habilidades pre-lectoras desde el nivel inicial (Bravo 
Valdivieso et al., 2006) y comprometer a la familia del estudiante con este propósito; asimismo, desarrollar métodos de enseñanza que, entre sus fines tenga, el privilegiar el desarrollo de las estrategias de metacomprensión lectora. Al hacerlo así, se mejoraría el rendimiento académico por las relaciones observadas entre estas dos variables (Quaas, Ascorra y Bertoglia, 2005), contribuyendo también a este objetivo el desarrollo de los procesos de atención y concentración desde los inicios de la vida escolar (Bazán, Roldán y Villaroel (2003).

Por otro lado, el no haber encontrado en la población estudiada un estilo de aprendizaje predominante coincide con la observación realizada en estudiantes de una Universidad Privada trujillana donde, de manera separada, en muestras de estudiantes de las áreas de letras y de ciencias, no se encontró algún estilo predominante; hallazgos que, de otra parte, contradicen otros que en mayoría coinciden en la existencia de algún estilo prevalente: asimilador y convergente en universitarios chilenos (Saavedra y Reynaldos, 2006); reflexivo, en universitarios chiclayanos de una Universidad Privada (Yacarini, 2005); divergente, en estudiantes de maestría de las universidades de San Marcos y Ricardo Palma (Delgado, 2004); divergente en estudiantes de Psicología de la Universidad Federico Villareal (Inga et al. 2001); convergente, divergente, asimilador y acomodador, según las carreras agrupadas por áreas en profesores mejicanos del Instituto Tecnológico de Monterrey (Polanco-Bueno, 1991), asimilador y convergente en estudiantes españoles y franceses de Psicología del Trabajo (Prieto, 1991), asimilador, en estudiantes de Psicología De La Universidad De San Marcos (Escurra, 1991).

Se realizó la comparación interuniversidades, se observó cómo los alumnos de la Universidad Nacional se mostraban más divergentes y asimiladores y con una capacidad de aprendizaje de conceptualización abstracta más elevada; y los de la Universidad Privada más convergentes y con capacidades de aprendizaje organización reflexiva y experiencia concreta más desarrolladas.

En consecuencia, caracteriza a los estudiantes de la Universidad Nacional el Estilo divergente (recepción y percepción de la información por Experiencia concreta - procesamiento de la información por Organización reflexiva). Su énfasis se encuentra en la imaginación y en los sentimientos, con capacidad para analizar situaciones concretas desde variadas posiciones y percibir las múltiples relaciones que se encuentran entre ellas, generando alternativas a estas con relativa facilidad. Asimismo, también los caracterizaría un Estilo Asimilador (percepción de la información por Conceptualización abstracta y procesamiento de la información por Organización reflexiva), tendiendo a agrupar las observaciones dentro de explicaciones integradas y modelos teóricos. El énfasis radica en las ideas, conceptos y teorías abstractas, con menor interés en el valor práctico. Por otro lado, caracteriza a los estudiantes de la Universidad Privada el Estilo convergente (recepción y percepción de la información por Conceptualización abstracta y procesamiento de la información por Experimentación Activa), tendiendo a funcionar mejor donde la deducción conduce a la respuesta correcta. Hacen énfasis en la aplicación práctica de las ideas, la toma de decisiones y la resolución de problemas. Los sujetos con este estilo prefieren situaciones no emotivas, tales como tareas técnicas en lugar de tareas sociales e impersonales; este estilo de aprendizaje es el característico de muchos ingenieros. Ya que este estudio no 
se diseñó para establecer las fuentes de los estilos o para determinar antes del ingreso cuáles eran los mismos, suponemos que las diferencias en los estilos de adquisición de conocimiento y en las modalidades o capacidades de aprendizaje entre los estudiantes de ambas universidades, se relacionarían con los contenidos y métodos de enseñanza de las carreras que estas ofrecen.

Finalmente, al no haberse comprobado la relación sustentada teóricamente de las estrategias de metacomprensión lectora con los estilos de aprendizaje, pensamos que se debe al bajo desarrollo de estas estrategias en la población estudiada (en términos absolutos, 535 estudiantes; en términos relativos, el $66 \%$ o 7 de cada 10 estudiantes se ubican en el nivel bajo global, y solamente 18 , el 4,6\% se sitúan en el nivel alto), en forma opuesta a los estilos de aprendizaje en los que no hay alguno que predomine (divergente, asimilador, convergente y acomodador); este bajo desarrollo ha producido una contracción de la dispersión, que se refleja en la no existencia de relación de ambas variables.

\section{CONCLUSIONES}

1. La muestra investigada posee un nivel global bajo en el desarrollo de estrategias de metacomprensión lectora.

2. La investigación indica que, según la universidad de procedencia, los estudiantes de la Universidad nacional se hallan en menor proporción en el nivel bajo, y en mayor proporción en el nivel medio en el uso de estrategias de metacomprensión lectora, en comparación con los estudiantes de la Universidad privada.

3. La investigación aporta datos que indican que los estudiantes de la Universidad Nacional poseen un mejor desarrollo de las estrategias metacomprensivas siguientes: predecir el contenido de un texto, verificar las predicciones, extraer conclusiones y autopreguntarse.

4. No se encontró evidencia en la muestra estudiada de que un estilo de aprendizaje predomine significativamente sobre los otros.

5. La investigación indica que, en los estudiantes de la Universidad nacional, es más frecuente el estilo divergente, seguido por el estilo asimilador; mientras que los estudiantes de la Universidad privada se inclinan más por el estilo convergente.

6. La investigación aporta datos específicos sobre las modalidades o capacidades de aprendizaje en cuanto a experiencia concreta y organización reflexiva, en donde los estudiantes de la Universidad privada tienen un mayor desarrollo; mientras que los estudiantes de la Universidad nacional lo evidencian en la capacidad de Conceptualización abstracta.

7. En la muestra estudiada, el nivel global de las estrategias de metacomprensión lectora y los estilos de aprendizaje son variables que no están asociadas. 


\section{REFERENCIAS BIBLIOGRÁFICAS}

8. Alonso, C., Gallego, D. y Money, P. (1997). Los estilos de aprendizaje, procedimientos de diagnóstico y mejora. Bilbao: Mensajero.

9. Alvarado, K. (2003). Los procesos metacognitivos: la metacomprensión y la actividad de la lectura. Revista Electrónica “Actualidades Investigativas en Educación Instituto de Investigación en Educación, Universidad de Costa Rica. Vol. 3, N. ${ }^{o} 2$. Recuperado el 2 de enero de 2008. Disponible en: http://www.erevistas.csic.es/portal/ listar_articulos.jsp?idAnio $=2003 \&$ idNum $=2 \& \mathrm{idVol}=3 \& \mathrm{idRevi}=84 \& \mathrm{idClas}=5$

10. Anaya, D. (2005). Efecto del resumen sobre la mejora de la Metacomprensión, la comprensión lectora y el rendimiento académico. Recuperado el 2 de enero de 2008 . Disponible en http://www.revistaeducacion.mec.es/re337/re337_14.pdfUniversidad Nacional de Educación a Distancia (UNED).

11. Bazán, Z.; Roldán, K. y Villaroel, M. (2003). Relación entre la capacidad de atención-concentración y el nivel de comprensión lectora en niños de cuarto y quinto grados de primaria de centros educativos estatales del distrito de La Molina. Tesis no publicada para optar el grado de magíster en Psicología. Unife. Lima, Perú.

12. Bravo Valdiviezo, L.; Villalon, M. y Orellana, E. (2006). Predictibilidad del rendimiento en la lectura: Una investigación de seguimiento entre primer y tercer año. Revista Latinoamericana de Psicología. Vol. 38, N. ${ }^{\circ}$ 1. Bogotá: Fundación Universitaria Konrad Lorenz, pp. 9-20.

13. Capella, J., Coloma, C., Manrique, L., Quevedo, E., Revilla, D., Tafur, R. y Vargas, J. (2003). Estilos de Aprendizaje. Lima: PUCP.

14. Condori, L. (2003). Aplicación de estrategias metacognitivas para mejorar la comprensión lectora en alumnos de cuarto grado " $b$ " de la institución educativa pública N. ${ }^{o} 70537$ del distrito de Cabanillas de la provincia de San Román del departamento de Puno. Lima: País.

15. Chrobak, R. (2000). La metacognición y las herramientas didácticas. Recuperado el 10 de enero de 2008. Disponible en: http://www.unrc.edu.ar/publicar/cde/05/ Chrobak.htm

16. Delgado, A. (2004). Relación entre los estilos de aprendizaje y los estilos de pensamiento en estudiantes de maestría considerando las especialidades profesionales y el tipo de universidad. Recuperado el 8 de marzo de 2008. Disponible en: http:// www.cybertesis.edu.pe/sisbib/2004/delgado_va/html/index-frames.html

17. Escurra, L. (1991). Adaptación del Inventario de Estilos de Aprendizaje de Kolb en Estudiantes de Psicología pertenecientes a diferentes universidades de Lima Metropolitana. Tesis no publicada para optar el Título Profesional de Psicólogo. Universidad Nacional Mayor de San Marcos, Lima, Perú.

18. Flavell, J. (1993). El desarrollo cognitivo. Madrid: Visor. 
19. Inga, J.; García, A.; Montes de Oca Serpa, J. y Capa, W. (2001). Estilos de Aprendizaje y Rendimiento académico en universitarios. Revista Científica Wiñay Yachay. Vol. 2, N. ${ }^{\circ}$ 1, pp. 93-98.

20. Irrazabal, N., Saux, G., Burín, D. y Lewón, J. (2004). Metacomprensión. Un estudio de comprensión lectora en estudiantes universitarios. Cuarto Congreso Nacional y Segundo Internacional de Investigación Educativa. Universidad Nacional del Comahue. Argentina. Recuperado el 18 de marzo de 2008. Disponible en: http:// face.uncoma.edu.ar/investigacion/congreso/articulos/area \%202/t139\%20-\%20 irrazabal \%20y\%20otros \%20-\%20ponencia.pdf

21. Kolb, D. A. (1976). Learning Style Inventory: Technical Manual. Boston: Mc Bier y Co.

22. Mateos, M. (2001). Metacognición y educación. Buenos Aires: Aique.

23. Meza, A. y Lazarte, C. (2007). Manual de estrategias para el aprendizaje autónomo y eficaz. Lima: Universidad Ricardo Palma, Editorial Universitaria.

24. Mialaret, G. (1972). Aprendizaje de la lectura. Madrid: Marova.

25. Plovnick, M.(1975). Primary Care Careers Choices and Medical Student Learning Styles. Journal of Medical Education. 50, 849-855.

26. Puente, A. (1994). Estilos de aprendizaje y enseñanza. Madrid: Cepe.

27. Quaas, C., Ascorra, P. y Bertoglia, L. (2005). Estrategias metacomprensivas lectoras y constructos asociados: en búsqueda de una relación. Psicoperspectivas. Revista de la Escuela de Psicología, Facultad de Filosofía y educación. Pontificia Universidad Católica de Valparaíso Vol. IV, pp. 77-90.

28. Saavedra, E. y Reynaldos, C. (2006). Caracterización cognitiva y emocional de los estudiantes de la Universidad Católica del Maule: Años 1999, 2001, 2003. Estudios Pedagógicos [online]. Vol. 32, N. ${ }^{\circ}$ 2, pp. 87-102. Recuperado el 10 Mayo de 2008. Disponible en: http://www.scielo.cl/scielo.php?script $=$ sci_arttex t\&pid =S071807052006000200005\&lng =es\&nrm =iso

29. Santibáñez, R. y Pisconte, M. (2003). Efectos del programa de reforzamiento de lectura orientado al desarrollo de habilidades metalectoras en los alumnos de estudios generales de la Universidad Nacional de Piura. Tesis para optar el grado de magíster en Psicología. Unife. Lima, Perú.

30. Schmitt, J. T. (1990). A questionnaire to measure children's awareness of strategic reading processes. The Reading Teacher. Vol. 43, N. ${ }^{\circ} 7$.

31. Torreblanca, A. (2001). ¿Cuán diferentes son los resultados de las pruebas de matemáticas y de lenguaje en Latinoamérica? Una mirada a través de la Laboratorio Americano de Evaluación de la Calidad Educacional. Crecer. Recuperado el 11 dejunio de 2007. Disponible en: http://www.minedu.gob.pe/web/el_ministerio/ el_ministerio/ PlaniEstra/umc/revista/revcrecer_02.pdf 
32. Yacarini, A. (2005). Estilos de aprendizaje y rendimiento académico en estudiantes de la Universidad Católica Santo Toribio de Mogrovejo, en Chiclayo, Perú. Recuperado el 20 de febrero de 2008. Disponible en: http://www.cienciaperu.org/eci2005i/ Libroresumenesaprobados2005i.htm 